\title{
ON REPRESENTATIONS OF QUANTUM GROUPS $U_{q}\left(f_{m}(K, H)\right)$
}

\author{
XIN TANG ${ }^{凶}$ and YUNGE XU
}

\author{
(Received 28 December 2007)
}

\begin{abstract}
We construct families of irreducible representations for a class of quantum groups $U_{q}\left(f_{m}(K, H)\right.$. First, we realize these quantum groups as hyperbolic algebras. Such a realization yields natural families of irreducible weight representations for $U_{q}\left(f_{m}(K, H)\right)$. Second, we study the relationship between $U_{q}\left(f_{m}(K, H)\right)$ and $U_{q}\left(f_{m}(K)\right)$. As a result, any finite-dimensional weight representation of $U_{q}\left(f_{m}(K, H)\right)$ is proved to be completely reducible. Finally, we study the Whittaker model for the center of $U_{q}\left(f_{m}(K, H)\right)$, and a classification of all irreducible Whittaker representations of $U_{q}\left(f_{m}(K, H)\right)$ is obtained.
\end{abstract}

2000 Mathematics subject classification: 17B10, 17B35, $17 \mathrm{~B} 37$.

Keywords and phrases: hyperbolic algebras, spectral theory, Whittaker model, quantum groups.

\section{Introduction}

Several generalizations (or deformations) of the quantized enveloping algebra $U_{q}\left(s l_{2}\right)$ have been extensively studied in [2, 7, 9, 17, 8]. Especially in [8], a general class of algebras $U_{q}(f(K))$ (similar to $U_{q}\left(s l_{2}\right)$ ) was introduced, and their finite-dimensional representations were studied. The representation theory of these algebras was further studied in [16] from the perspectives of both spectral theory [14] and the Whittaker model [10]. In [17], as generalizations of the algebras $U_{q}(f(K))$, another general class of algebras $U_{q}(f(K, H))$ was introduced and studied. Note that the Drinfeld quantum double of the positive part of the quantized enveloping algebra $U_{q}\left(s l_{2}\right)$ studied in [7], or equivalently the two-parameter quantum groups $U_{r, s}\left(s l_{2}\right)$ studied in [2], is a special case of the algebra $U_{q}(f(K, H))$. The condition on the parameter Laurent polynomial $f(K, H) \in \mathbb{C}\left[K^{ \pm 1}, H^{ \pm 1}\right]$ for the existence of a Hopf algebra structure on $U_{q}(f(K, H))$ was determined, and finite dimensional irreducible representations were explicitly constructed as quotients of highest weight representations in [17]. This class of algebras provides a family of quantum groups in the sense of Drinfeld [4]. In particular, $U_{q}\left(f_{m}(K, H)\right)$ are quantum groups for

The second author was partially supported by NSFC, under grant 10501010 .

(C) 2008 Australian Mathematical Society 0004-9727/08 \$A2.00+0.00 


$$
f_{m}(K, H)=\frac{K^{m}-H^{m}}{q-q^{-1}}, \quad m \in \mathbb{N} .
$$

In this paper, we study the irreducible representations of these quantum groups $U_{q}\left(f_{m}(K, H)\right)$. Although most of the results in this paper hold for the algebras $U_{q}(f(K, H))$ with general parameters, the calculations are more complicated.

It is not surprising that these quantum groups share many similar properties with the two-parameter quantum groups $U_{r, s}\left(s l_{2}\right)$. However, it may be useful to get more explicit information on the representation theory of these quantum groups. In the first part of this paper, we study the irreducible weight representations (which are not necessarily finite-dimensional) of $U_{q}\left(f_{m}(K, H)\right)$ from the viewpoint of spectral theory. Namely, we realize these quantum groups as hyperbolic algebras, then apply the general results on hyperbolic algebras established in [14] to construct natural families of irreducible weight representations for $U_{q}\left(f_{m}(K, H)\right)$. Such an approach yields the highest weight, the lowest weight and weight irreducible representations for $U_{q}\left(f_{m}(K, H)\right)$.

We denote by $f_{m}(K)$ the Laurent polynomial $\left(K^{m}-K^{-m}\right) /\left(q-q^{-1}\right)$. Note that there is a close relationship between the representation theory of $U_{q}\left(f_{m}(K)\right)$ and that of $U_{q}\left(f_{m}(K, H)\right)$. We investigate this relationship following the idea in [7]. As an application, we obtain some nice results on the category of all weight representations of $U_{q}\left(f_{m}(K, H)\right)$. In particular, we show that the category of all weight representations of $U_{q}\left(f_{m}(K, H)\right)$ is equivalent to the product of the category of weight representations of $U_{q}\left(f_{m}(K)\right)$ with $\mathbb{C}^{*}$ as a tensor category. Combined with a result proved for $U_{q}\left(f_{m}(K)\right)$ in [8], we show that any finite dimensional weight representation of $U_{q}\left(f_{m}(K, H)\right)$ is completely reducible.

Finally, we study the Whittaker model for the center of these quantum groups. We prove that any Whittaker representation is irreducible if and only if it admits a central character. This criterion gives a complete classification of all irreducible Whittaker representations of $U_{q}\left(f_{m}(K, H)\right)$.

The paper is organized as follows. In Section 2, we recall the definitions of $U_{q}(f(K))$ and $U_{q}(f(K, H))$, and some basic facts about them from [17, 8]. In Section 3, we recall some basic facts about spectral theory and hyperbolic algebras from [14]. Then we realize $U_{q}(f(K, H))$ as hyperbolic algebras, and construct natural families of irreducible weight representations for $U_{q}\left(f_{m}(K, H)\right)$. In Section 4, we study the relationship between $U_{q}\left(f_{m}(K)\right)$ and $U_{q}\left(f_{m}(K, H)\right)$ from the perspective of representation theory. In Section 5, we construct the Whittaker model for the center of $U_{q}\left(f_{m}(K, H)\right)$, and study the Whittaker representations of $U\left(f_{m}(K, H)\right)$. We obtain a classification of all irreducible Whittaker representations.

\section{The algebras $U_{q}(f(K, H))$}

Let $\mathbb{C}$ be the field of complex numbers and $0 \neq q \in \mathbb{C}$ such that $q^{2} \neq 1$. It is well known that the quantized enveloping algebra $U_{q}\left(s l_{2}\right)$ corresponding to the simple 
Lie algebra $s l_{2}$ is the associative $\mathbb{C}$-algebra generated by $E, F, K^{ \pm 1}$ subject to the following relations:

$$
\begin{gathered}
K E=q^{2} E K, \quad K F=q^{-2} F K, \quad K K^{-1}=K^{-1} K=1, \\
E F-F E=\frac{K-K^{-1}}{q-q^{-1}} .
\end{gathered}
$$

Note that $U_{q}\left(s l_{2}\right)$ is a Hopf algebra with a Hopf algebra structure defined as follows:

$$
\begin{gathered}
\Delta(E)=E \otimes 1+K \otimes E, \quad \Delta(F)=F \otimes K^{-1}+1 \otimes F \\
\varepsilon(E)=0=\varepsilon(F), \quad \varepsilon(K)=1=\varepsilon\left(K^{-1}\right) ; \\
s(E)=-K^{-1} E, \quad s(F)=-F K, \quad s(K)=K^{-1} .
\end{gathered}
$$

As generalizations of $U_{q}\left(s l_{2}\right)$, a class of algebras $U_{q}(f(K))$ parameterized by Laurent polynomials $f(K) \in \mathbb{C}\left[K, K^{-1}\right]$ was introduced in [8]. For the reader's convenience, we recall their definition here.

Definition 2.1 [8]. For any Laurent polynomial $f(K) \in \mathbb{C}\left[K, K^{-1}\right]$, $U_{q}(f(K))$ is defined to be the $\mathbb{C}$-algebra generated by $E, F, K^{ \pm 1}$ subject to the following relations:

$$
\begin{gathered}
K E=q^{2} E K, \quad K F=q^{-2} F K \\
K K^{-1}=K^{-1} K=1 ; \\
E F-F E=f(K) .
\end{gathered}
$$

The ring theoretic properties and finite-dimensional representations were first studied in detail in [8]. We state some of these results here without proof. First of all, for the Laurent polynomials $f(K)=a\left(K^{m}-K^{-m}\right)$ where $a \in \mathbb{C}^{*}$ and $m \in \mathbb{N}$, the algebras $U_{q}(f(K))$ have a Hopf algebra structure. In particular, we have the following result from [8].

Proposition 2.2 [8, Proposition 3.3]. Assume $f(K)$ is a nonzero Laurent polynomial in $\mathbb{C}\left[K, K^{-1}\right]$. Then the noncommutative algebra $U_{q}(f(K))$ is a Hopf algebra such that $K, K^{-1}$ are group-like elements, and $E, F$ are skew primitive elements if and only if $f(K)=a\left(K^{m}-K^{-m}\right)$ with $m=t-s$ and the following conditions are satisfied:

$$
\begin{gathered}
\Delta(K)=K \otimes K, \quad \Delta\left(K^{-1}\right)=K^{-1} \otimes K^{-1} ; \\
\Delta(E)=E^{s} \otimes E+E \otimes K^{t}, \quad \Delta(F)=K^{-t} \otimes F+F \otimes K^{-s} ; \\
\varepsilon(K)=\varepsilon\left(K^{-1}\right)=1, \quad \varepsilon(E)=\varepsilon(F)=0 ; \\
S(K)=K^{-1}, \quad S\left(K^{-1}\right)=K ; \\
S(E)=-K^{-s} E K^{-t}, \quad S(F)=-K^{t} F K^{s} .
\end{gathered}
$$


For the case $f_{m}(K)=\left(K^{m}-K^{-m}\right) /\left(q-q^{-1}\right)$ for $m \in \mathbb{N}$ and $q$ not being a root of unity, the finite-dimensional irreducible representations were proved to be highest weight and constructed explicitly in [8]. Furthermore, any finite dimensional representations are completely reducible as stated in the following theorem from [8].

THEOREM 2.3 [8, Theorem 4.17]. With the above assumption for $f_{m}(K)$ and $q$, any finite dimensional representation $V$ of $U_{q}\left(f_{m}(K)\right)$ is completely reducible.

REMARK 2.4. The representation theory of $U_{q}\left(f_{m}(K)\right)$ was studied further from the points of view of spectral theory and the Whittaker model in [16], where more families of interesting irreducible representations were constructed.

As generalizations of the algebras $U_{q}(f(K))$, another general class of algebras parameterized by Laurent polynomials $f(K, H) \in \mathbb{C}\left[K^{ \pm 1}, H^{ \pm 1}\right]$ was introduced and studied in [17]. First, let us recall the definition of $U_{q}(f(K, H))$.

Definition 2.5 [17]. Let $f(K, H) \in \mathbb{C}\left[K^{ \pm 1}, H^{ \pm 1}\right]$ be a Laurent polynomial, and $U_{q}(f(K, H))$ is defined to be the $\mathbb{C}$-algebra generated by $E, F, K^{ \pm 1}, H^{ \pm 1}$ subject to the following relations:

$$
\begin{gathered}
K E=q^{2} E K, \quad K F=q^{-2} F K, \\
H E=q^{-2} E H, \quad H F=q^{2} F H, \\
K K^{-1}=K^{-1} K=1=H H^{-1}=H^{-1} H, \quad K H=H K, \\
E F-F E=f(K, H) .
\end{gathered}
$$

It is easy to see that the Drinfeld quantum double of the positive part of $U_{q}\left(s l_{2}\right)$ [7] or the two-parameter quantum group $U_{r, s}\left(s l_{2}\right)$ [2] is a special case of the algebra $U_{q}(f(K, H))$. The condition on the parameter $f(K, H)$ for the existence of a Hopf algebra structure on $U_{q}(f(K, H))$ was determined, and finite-dimensional irreducible representations were constructed explicitly as quotients of highest weight representations in [17]. In addition, a counter example was also constructed in [17] to show that not all finite-dimensional representations are completely reducible. So it would be interesting to know what kind of finite-dimensional representations are completely reducible. We shall address this question in Section 4.

\section{Hyperbolic algebras and their representations}

In this section, we realize $U_{q}(f(K, H))$ as hyperbolic algebras and apply the methods in spectral theory as developed in [14] to construct irreducible weight representations of $U_{q}\left(f_{m}(K, H)\right)$. For the reader's convenience, we need to recall a little bit of background about spectral theory and hyperbolic algebras from [14].

3.1. Preliminaries on spectral theory Spectral theory of abelian categories was first started by Gabriel in [5]. He defined the injective spectrum of any noetherian Grothendieck category. This spectrum consists of isomorphism classes of indecomposable injective objects. If $R$ is a commutative noetherian ring, then 
the spectrum of the category of all $R$-modules is isomorphic to the prime spectrum $\operatorname{Spec}(R)$ of $R$. And one can reconstruct any noetherian commutative scheme $\left(X, O_{X}\right)$ using the spectrum of the category of quasi-coherent sheaves of modules on $X$. The spectrum of any abelian category was later on defined by Rosenberg in [14]. This spectrum works for any abelian category. Via this spectrum, one can reconstruct any quasi-separated and quasi-compact commutative scheme $\left(X, O_{X}\right)$ via the spectrum of the category of quasi-coherent sheaves of modules on $X$.

To proceed, we review some basic notions and facts about the spectrum of any abelian category. First of all, we recall the definition of the spectrum of any abelian category, then we explain its applications in representation theory. We refer the reader to [14] for more details.

Let $C_{X}$ be an abelian category and $M, N \in C_{X}$ be any two objects; we say that $M \succ N$ if and only if $N$ is a sub-quotient of the direct sum of finitely many copies of $M$. It is easy to verify that $\succ$ is a pre-order. We say that $M \approx N$ if and only if $M \succ N$ and $N \succ M$. It is obvious that $\approx$ is an equivalence. Let $\operatorname{Spec}(X)$ be the family of all nonzero objects $M \in C_{X}$ such that, for any nonzero sub-object $N$ of $M, N \succ M$.

Definition 3.1 [14]. The spectrum of any abelian category is defined to be:

$$
\operatorname{Spec}(X)=\operatorname{Spec}(X) / \approx .
$$

Although spectral theory is more important for the purpose of noncommutative algebraic geometry, it has nice applications to representation theory. The notion of the spectrum has a natural analogue of the Zariski topology. Under certain mild finiteness conditions, its closed points are in a one-to-one correspondence with the irreducible objects of the category. In particular, this is true for the category of representations of an algebra. To study irreducible representations, one can study the spectrum of the category of all representations, then single out closed points of the spectrum with respect to the associated topology.

3.2. The left spectrum of a ring If $C_{X}$ is the category $A$-mod of left modules over a ring $A$, then it is sometimes convenient to express the points of $\operatorname{Spec}(X)$ in terms of left ideals of the ring $A$. In order to do so, the left spectrum $\operatorname{Spec}_{l}(A)$ was defined in [14], which is by definition the set of all left ideals $p$ of $A$ such that $A / p$ is an object of $\operatorname{Spec}(X)$. The relation $\succ$ on $A$-mod induces a specialization relation among left ideals, in particular, the $\operatorname{specialization}$ relation on $\operatorname{Spec}_{l}(A)$. Namely, $A / m \succ A / n$ if and only if there exists a finite subset $x$ of elements of $A$ such that the ideal $(n: x)=\{a \in A \mid a x \subset n\}$ is contained in $m$. Following [14], we denote this by $n \leq m$. Note that the relation $\leq$ is just the inclusion if $n$ is a two-sided ideal. In particular, it is the inclusion if the ring $A$ is commutative. The map which assigns to

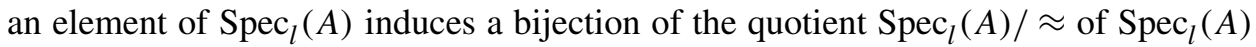
by the equivalence relation associated with $\leq$ onto $\operatorname{Spec}(X)$. From now on, we shall not distinguish $\operatorname{Spec}_{l}(A) / \approx \operatorname{from} \operatorname{Spec}(X)$ and will express results in terms of the left spectrum. 
3.3. Hyperbolic algebra $\boldsymbol{R}\{\boldsymbol{\xi}, \boldsymbol{\theta}\}$ and its spectrum Hyperbolic algebras are studied by Rosenberg in [14] and by Bavula under the name of Generalized Weyl algebras in [1]. Hyperbolic algebra structure is very convenient for the construction of points of the spectrum. As an application of spectral theory to representation theory, points of the left spectrum have been constructed for hyperbolic algebras in [14]. Many 'small' algebras including the first Weyl algebra $A_{1}$, the enveloping algebra $U\left(s l_{2}\right)$, and their quantized versions or deformations, are hyperbolic algebras. We shall review some basic facts about hyperbolic algebras and two important construction theorems from [14].

Let $\theta$ be an automorphism of a commutative algebra $R$; and let $\xi$ be an element of $R$.

Definition 3.2. The hyperbolic algebra $R\{\theta, \xi\}$ is defined to be the $R$-algebra generated by $x, y$ subject to the following relations:

$$
x y=\xi, \quad y x=\theta^{-1}(\xi)
$$

and

$$
x a=\theta(a) x, \quad y a=\theta^{-1}(a) y
$$

for any $a \in R . R\{\theta, \xi\}$ is called a hyperbolic algebra over $R$.

Let $C_{X}=C_{R\{\theta, \xi\}}$ be the category of modules over $R\{\theta, \xi\}$. We denote by $\operatorname{Spec}(X)$ the spectrum of $C_{X}$. Points of the left spectrum of hyperbolic algebras are studied in [14], and, in particular, we have the following construction theorems from [14].

Theorem 3.3 [14, Theorem 3.2.2]. (1) Let $P \in \operatorname{Spec}(R)$, and assume that the orbit of $P$ under the action of the $\theta$ is infinite.

(a) If $\theta^{-1}(\xi) \in P$, and $\xi \in P$, then the left ideal

$$
P_{1,1}:=P+R\{\theta, \xi\} x+R\{\theta, \xi\} y
$$

is a two-sided ideal from $\operatorname{Spec}_{l}(R\{\theta, \xi\})$.

(b) If $\theta^{-1}(\xi) \in P, \theta^{i}(\xi) \notin P$ for $0 \leq i \leq n-1$, and $\theta^{n}(\xi) \in P$, then the left ideal

$$
P_{1, n+1}:=R\{\theta, \xi\} P+R\{\theta, \xi\} x+R\{\theta, \xi\} y^{n+1}
$$

belongs to $\operatorname{Spec}_{l}(R\{\theta, \xi\})$.

(c) If $\theta^{i}(\xi) \notin P$ for $i \geq 0$, and $\theta^{-1}(\xi) \in P$, then

$$
P_{1, \infty}:=R\{\theta, \xi\} P+R\{\theta, \xi\} x
$$

belongs to $\operatorname{Spec}_{l}(R\{\theta, \xi\})$.

(d) If $\xi \in P$, and $\theta^{-i}(\xi) \notin P$, for all $i \geq 1$, then the left ideal

$$
P_{\infty, 1}:=R\{\theta, \xi\} P+R\{\theta, \xi\} y
$$

belongs to $\operatorname{Spec}_{l}(R\{\theta, \xi\})$.

(2) If the ideal $P$ in (b), (c) or (d) is maximal, then the corresponding left ideal of $\operatorname{Spec}_{l}(R\{\theta, \xi\})$ is maximal. 
(3) Every left ideal $Q \in \operatorname{Spec}_{l}(R\{\theta, \xi\})$, such that $\theta^{v}(\xi) \in Q$ for $a v \in \mathbb{Z}$, is equivalent to one left ideal as defined above uniquely from a prime ideal $P \in \operatorname{Spec}(R)$. The latter means that if $P$ and $P^{\prime}$ are two prime ideals of $R$, and $(\alpha, \beta)$ and $(\nu, \mu)$ take values $(1, \infty),(\infty, 1),(\infty, \infty)$ or $(1, n)$, then $P_{\alpha, \beta}$ is equivalent to $P_{v, \mu}^{\prime}$ if and only if $\alpha=v, \beta=\mu$ and $P=P^{\prime}$.

Theorem 3.4 [14, Proposition 3.2.3]. (1) Let $P \in \operatorname{Spec}(R)$ be a prime ideal of $R$ such that $\theta^{i}(\xi) \notin P$ for $i \in \mathbb{Z}$ and $\theta^{i}(P)-P \neq \varnothing$ for $i \neq 0$, then $P_{\infty, \infty}$ $=R\{\xi, \theta\} P \in \operatorname{Spec}_{l}(R\{\xi, \theta\})$.

(2) Moreover, if $\mathbf{P}$ is a left ideal of $R\{\theta$, $\xi\}$ such that $\mathbf{P} \cap R=P$, then $\mathbf{P}=P_{\infty, \infty}$. In particular, if $P$ is a maximal ideal, then $P_{\infty, \infty}$ is a maximal left ideal.

(3) If a prime ideal $P^{\prime} \subset R$ is such that $P_{\infty, \infty}=P_{\infty, \infty}^{\prime}$, then $P^{\prime}=\theta^{n}(P)$ for some integer $n$. Conversely, $\theta^{n}(P)_{\infty, \infty}=P_{\infty, \infty}$ for any $n \in \mathbb{Z}$.

3.4. Realize $U_{\boldsymbol{q}}(\boldsymbol{f}(\boldsymbol{K}, \boldsymbol{H}))$ as hyperbolic algebras Let $R$ be the sub-algebra of $U_{q}(f(K, H))$ generated by $E F, K^{ \pm 1}, H^{ \pm 1}$, then $R$ is a commutative algebra. We define an algebra automorphism $\theta: R \longrightarrow R$ of $R$ by setting

$$
\begin{gathered}
\theta(E F)=E F+f(\theta(K), \theta(H)), \\
\theta\left(K^{ \pm 1}\right)=q^{\mp 2} K^{ \pm 1}, \\
\theta\left(H^{ \pm 1}\right)=q^{ \pm 2} H^{ \pm 1}
\end{gathered}
$$

It is easy to see that $\theta$ extends to an algebra automorphism of $R$. Furthermore, we have the following lemma.

LEMMA 3.5. The following identities hold:

$$
\begin{aligned}
E(E F) & =\theta(E F) E, \\
F(E F) & =\theta^{-1}(E F) F, \\
E K & =\theta(K) E, \\
F K & =\theta^{-1}(K) F, \\
E H & =\theta(H) E, \\
F H & =\theta^{-1}(H) F .
\end{aligned}
$$

PROOF. We only verify the first one, and the rest of them can be checked similarly.

$$
\begin{aligned}
E(E F) & =E(F E+f(K, H)) \\
& =(E F) E+E f(K, H) \\
& =(E F) E+f(\theta(K), \theta(H)) E \\
& =(E F+f(\theta(K), \theta(H))) E \\
& =\theta(E F) E .
\end{aligned}
$$

So we are done.

From Lemma 3.5, we have the following result. 
Proposition 3.6. $U_{q}(f(K, H))=R\{\xi=E F, \theta\}$ is a hyperbolic algebra with $R$ and $\theta$ defined as above.

It easy to see that we have the following corollary.

Corollary 3.7 [17, Proposition 2.5]. $U_{q}(f(K, H))$ is noetherian domain of GK-dimension 4.

3.5. Families of irreducible weight representations of $\boldsymbol{U}_{q}\left(f_{m}(\boldsymbol{K}, \boldsymbol{H})\right)$ Now we can apply the above construction theorems to the case of $U_{q}\left(f_{m}(K, H)\right)$, and construct families of irreducible weight representations of $U_{q}\left(f_{m}(K, H)\right)$.

Given $\alpha, \beta, \gamma \in \mathbb{C}$, we denote by

$$
M_{\alpha, \beta, \gamma}=(\xi-\alpha, K-\beta, H-\gamma) \subset R
$$

the maximal ideal of $R$ generated by $\xi-\alpha, K-\beta, H-\gamma$. We have the following lemma.

LEMMA 3.8. $\theta^{n}\left(M_{\alpha, \beta, \gamma}\right) \neq M_{\alpha, \beta, \gamma}$ for any $n \geq 1$. In particular, $M_{\alpha, \beta, \gamma}$ has an infinite orbit under the action of $\theta$.

PROOF. We have

$$
\begin{aligned}
\theta^{n}(K-\beta) & =\left(q^{-2 n} K-\beta\right) \\
& =q^{-2 n}\left(K-q^{2 n} \beta\right)
\end{aligned}
$$

Since $q$ is not a root of unity, $q^{2 n} \neq 1$ for any $n \neq 0$. So we have $\theta^{n}\left(M_{\alpha, \beta, \gamma}\right) \neq M_{\alpha, \beta, \gamma}$ for any $n \geq 1$.

Now we construct all irreducible weight representations of $U_{q}\left(f_{m}(K, H)\right)$ with $f_{m}(K, H)=\left(K^{m}-H^{m}\right) /\left(q-q^{-1}\right), m \in \mathbb{N}$.

First of all, another lemma is in order.

LEMMA 3.9. For $n \geq 0$, we have the following formulae.

$$
\theta^{n}(E F)=E F+\frac{1}{q-q^{-1}}\left(\frac{q^{-2 m}\left(1-q^{-2 n m}\right)}{1-q^{-2 m}} K^{m}-\frac{q^{2 m}\left(1-q^{2 n m}\right)}{1-q^{2 m}} H^{m}\right)
$$

$$
\theta^{-n}(E F)=E F-\frac{1}{q-q^{-1}}\left(\frac{1-q^{2 n m}}{1-q^{2 m}} K^{m}-\frac{1-q^{-2 n m}}{1-q^{-2 m}} H^{m}\right) .
$$

PROOF. For $n \geq 0$,

$$
\begin{aligned}
\theta^{n}(E F)= & E F+\frac{1}{q-q^{-1}}\left(\left(q^{-2 m}+\cdots+q^{-2 n m}\right) K^{m}\right. \\
& \left.\quad-\left(q^{2 m}+\cdots+q^{2 n m}\right) H^{m}\right) \\
= & E F+\frac{1}{q-q^{-1}}\left(\frac{q^{-2 m}\left(1-q^{-2 n m}\right)}{1-q^{-2 m}} K^{m}-\frac{q^{2 m}\left(1-q^{2 n m}\right)}{1-q^{2 m}} H^{m}\right) .
\end{aligned}
$$

The second statement can be proved similarly. 
THEOREM 3.10. Let $P=M_{\alpha, \beta, \gamma}$, then we have the following formulae.

(1) If $\alpha=\left(\beta^{m}-\gamma^{m}\right) /\left(q-q^{-1}\right),(\beta / \gamma)^{m}=q^{2 m n}$ for some $n \geq 0$, then $\theta^{n}(\xi)$ $\in M_{\alpha, \beta, \gamma}$ and $\theta^{-1}(\xi) \in M_{\alpha, \beta, \gamma}$, thus $U_{q}\left(f_{m}(K, H)\right) / P_{1, n+1}$ is a finitedimensional irreducible representation of $U_{q}\left(f_{m}(K, H)\right)$.

(2) If $\alpha=\left(\beta^{m}-\gamma^{m}\right) /\left(q-q^{-1}\right)$ and $(\beta / \gamma)^{m} \neq q^{2 m n}$ for all $n \geq 0$, then $U_{q}\left(f_{m}(K, H)\right) / P_{1, \infty}$ is an infinite-dimensional irreducible representation of $U_{q}\left(f_{m}(K, H)\right)$.

(3) If $\alpha=0$ and

$$
0 \neq \frac{1}{q-q^{-1}}\left(\frac{1-q^{2 n m}}{1-q^{2 m}} \beta^{m}-\frac{1-q^{-2 n m}}{1-q^{-2 m}} \gamma^{m}\right)
$$

for any $n \geq 1$, then $U_{q}\left(f_{m}(K, H)\right) / P_{\infty, 1}$ is an infinite-dimensional irreducible representation of $U_{q}\left(f_{m}(K, H)\right)$.

Proof. Since $\theta^{-1}(\xi)=\xi-\left(K^{m}-H^{m}\right) /\left(q-q^{-1}\right)$, thus $\theta^{-1}(\xi) \in M_{\alpha, \beta, \gamma}$ if and only if $\alpha=\left(\beta^{m}-\gamma^{m}\right) /\left(q-q^{-1}\right)$. Now by the proof of Lemma 3.9,

$$
\begin{aligned}
\theta^{n}(\xi)=\xi+ & \frac{1}{q-q^{-1}}\left(\left(q^{-2 m}+\cdots+q^{-2 n m}\right) K^{m}\right. \\
& \left.-\left(q^{2 m}+\cdots+q^{2 n m}\right) H^{m}\right) \\
=\xi & +\frac{1}{q-q^{-1}}\left(\frac{q^{-2 m}\left(1-q^{-2 n m}\right)}{1-q^{-2 m}} K^{m}-\frac{q^{2 m}\left(1-q^{2 n m}\right)}{1-q^{2 m}} H^{m}\right) .
\end{aligned}
$$

Hence $\theta^{n}(\xi) \in M_{\alpha, \beta, \gamma}$ if and only if

$$
\begin{aligned}
0 & =\alpha+\frac{1}{q-q^{-1}}\left(\left(q^{-2 m}+\cdots+q^{-2 n m}\right) \beta^{m}-\left(q^{2 m}+\cdots+q^{2 n m}\right) \gamma^{m}\right) \\
& =\alpha+\frac{1}{q-q^{-1}}\left(\frac{q^{-2 m}\left(1-q^{-2 n m}\right)}{1-q^{-2 m}} \beta^{m}-\frac{q^{2 m}\left(1-q^{2 n m}\right)}{1-q^{2 m}} \gamma^{m}\right) .
\end{aligned}
$$

Hence when $\alpha=\left(\beta^{m}-\gamma^{m}\right) /\left(q-q^{-1}\right),(\beta / \gamma)^{m}=q^{2 m n}$ for some $n \geq 0$,

$$
\theta^{n}(\xi) \in M_{\alpha, \beta, \gamma}, \theta^{-1}(\xi) \in M_{\alpha, \beta, \gamma} .
$$

Thus by Theorem 3.3, $U_{q}\left(f_{m}(K, H)\right) / P_{1, n+1}$ is a finite dimensional irreducible representation of $U_{q}\left(f_{m}(K, H)\right)$. So we have already proved the first statement; the rest of the statements can be similarly proved.

REMARK 3.11. The representations we constructed in Theorem 3.10 exhaust all finite-dimensional irreducible weight representations, the highest weight irreducible representations and the lowest weight irreducible representations of $U_{q}\left(f_{m}(K, H)\right)$.

REMARK 3.12. Finite-dimensional irreducible weight representations have been constructed in [17] as quotients of highest weight representations. And a counter 
example has also been constructed in [17] to indicate that not all finite-dimensional representations are completely reducible.

Apply the second construction theorem, we have the following theorem.

THEOREM 3.13. Let $P=M_{\alpha, \beta, \gamma}$. If

$$
\alpha \neq-\frac{1}{q-q^{-1}}\left(\frac{q^{-2 m}\left(1-q^{-2 n m}\right)}{1-q^{-2 m}} \beta^{m}-\frac{q^{2 m}\left(1-q^{2 n m}\right)}{1-q^{2 m}} \gamma^{m}\right)
$$

for any $n \geq 0$ and

$$
\alpha \neq \frac{1}{q-q^{-1}}\left(\frac{1-q^{2 n m}}{1-q^{2 m}} \beta^{m}-\frac{1-q^{-2 n m}}{1-q^{-2 m}} \gamma^{m}\right)
$$

for any $n \geq 1$, then $U_{q}\left(f_{m}(K, H)\right) / P_{\infty, \infty}$ is an infinite-dimensional irreducible weight representation of $U_{q}\left(f_{m}(K, H)\right)$.

PROOF. The proof is very similar to that of Theorem 3.10; we shall omit it here.

COROLlary 3.14. The representations constructed in Theorems 3.10 and 3.13 exhaust all irreducible weight representations of $U_{q}\left(f_{m}(K, H)\right)$.

PROOF. It follows directly from Theorems 3.3-3.13.

\section{The relationship between $U_{q}\left(f_{m}(K)\right)$ and $U_{q}\left(f_{m}(K, H)\right)$}

Recall that we denote by $f_{m}(K, H)$ the polynomial $\left(K^{m}-H^{m}\right) /\left(q-q^{-1}\right)$, and by $f_{m}(K)$ the Laurent polynomial $\left(K^{m}-K^{-m}\right) /\left(q-q^{-1}\right)$. We compare the quantum groups $U_{q}\left(f_{m}(K)\right)$ and $U_{q}\left(f_{m}(K, H)\right)$. As a result, we prove that any finitedimensional weight representation of $U_{q}\left(f_{m}(K, H)\right)$ is completely reducible.

First of all, it is easy to see that we have the following lemma generalizing the situation in [7].

LeMMA 4.1. The map which sends $E$ to $E, F$ to $F, K^{ \pm 1}$ to $K^{ \pm 1}$, and $H^{ \pm 1}$ to $K^{\mp 1}$ extends to a unique surjective Hopf algebra homomorphism $\pi: U_{q}\left(f_{m}(K, H)\right)$ $\longrightarrow U_{q}\left(f_{m}(K)\right)$.

PROOF. Note that both $U_{q}\left(f_{m}(K)\right)$ and $U_{q}\left(f_{m}(K, H)\right)$ are Hopf algebras under the assumption on $f_{m}(K)$ and $f_{m}(K, H)$. Since the kernel of $\pi$ is generated by $K-H^{-1}$, it is a Hopf ideal of $U_{q}\left(f_{m}(K, H)\right)$. So we are done.

Our goal in this section is to describe those representations $M$ of $U_{q}\left(f_{m}(K, H)\right)$ such that $\operatorname{End}_{U_{q}\left(f_{m}(K, H)\right)}(M)=\mathbb{C}$. Since $K H$ is in the center and invertible, it acts on these representations by a nonzero scalar. As in [7], for each $z \in \mathbb{C}^{*}$, we define a $\mathbb{C}$-algebra homomorphism $\pi_{z}: U_{q}\left(f_{m}(K, H)\right) \longrightarrow U_{q}\left(f_{m}(K)\right)$ as follows:

$$
\begin{gathered}
\pi_{z}(E)=z^{m / 2} E, \quad \pi_{z}(F)=F ; \\
\pi_{z}(K)=z^{\frac{1}{2}} K, \quad \pi_{z}(H)=z^{\frac{1}{2}} K^{-1} .
\end{gathered}
$$


It is easy to see that $\pi_{z}$ is an algebra epimorphism with the kernel of $\pi_{z}$ being a two-sided ideal generated by $K H-z$. But they may not necessarily be a Hopf algebra homomorphism unless $z=1$.

Let $M$ be a representation of $U_{q}\left(f_{m}(K, H)\right)$. As in [7], we have the following lemma.

Lemma 4.2. Suppose that $\operatorname{End}_{U_{q}\left(f_{m}(K, H)\right)}(M)=\mathbb{C}$. Then there exists a unique $z \in \mathbb{C}^{*}$ such that $M$ is the pullback of a representation of $U_{q}\left(f_{m}(K)\right)$ through a $\pi_{z}$ as defined above. In particular, any such irreducible representation of $U_{q}\left(f_{m}(K, H)\right)$ is the pullback of an irreducible representation of $U_{q}\left(f_{m}(K)\right)$ through the algebra homomorphism $\pi_{z}$ for some $z \in \mathbb{C}^{*}$.

We use the notation in [7]. Let $M$ be a representation of $U_{q}\left(f_{m}(K)\right)$, and we denote by $M_{z}$ the representation of $U_{q}\left(f_{m}(K, H)\right)$ obtained as the pullback of $M$ via $\pi_{z}$. Let $\varepsilon_{z}$ be the one-dimensional representation of $U_{q}\left(f_{m}(K, H)\right)$ which is defined by mapping the generators of $U_{q}\left(f_{m}(K, H)\right)$ as follows:

$$
\begin{gathered}
\varepsilon_{z}(E)=\varepsilon_{z}(F)=0 ; \\
\varepsilon_{z}(K)=z^{\frac{1}{2}}, \quad \varepsilon_{z}(H)=z^{\frac{1}{2}} .
\end{gathered}
$$

Then we have the following similar lemma as in [7].

LEMMA 4.3. Let $0 \neq z \in \mathbb{C}$, and $M$ be a representation of $U_{q}\left(f_{m}(K)\right)$. Then $M_{z} \cong \varepsilon_{z}$ $\otimes M_{1} \cong M_{1} \otimes \varepsilon_{z}$. In particular, if $0 \neq z^{\prime} \in \mathbb{C}$, and $N$ is another representation of $U_{q}\left(f_{m}(K)\right)$, then

$$
M_{z} \otimes N_{z^{\prime}} \cong(M \otimes N)_{z z^{\prime}}
$$

PROOF. The proof is straightforward.

Let $M$ be a representation of $U_{q}\left(f_{m}(K, H)\right)$. We say $M$ is a weight representation if $H$ and $K$ are acting on $M$ semisimply. Let $\mathfrak{C}$ be the category of all weight representations of $U_{q}\left(f_{m}(K)\right)$ and $\tilde{\mathfrak{C}}$ be the category of all weight representations of $U_{q}\left(f_{m}(K, H)\right)$. Let $\mathbb{C}^{*}$ be the tensor category associated to the multiplicative group $\mathbb{C}^{*}$, then we have the following result.

THEOREM 4.4. The category $\tilde{\mathfrak{C}}$ is equivalent to the direct product of the categories $\mathfrak{C}$ and $\mathbb{C}^{*}$ as a tensor category.

PROOF. The proof is similar to the one in [7], and we refer the reader to [7] for more details.

COROLlary 4.5. Any finite-dimensional weight representation of $U_{q}\left(f_{m}(K, H)\right)$ is completely reducible.

PROOF. This follows from the above theorem and the fact that any finite dimensional representation of $U_{q}\left(f_{m}(K)\right)$ is completely reducible (as is proved in [8]). 
COROLlary 4.6. The tensor product of any two finite-dimensional weight representations of $U_{q}\left(f_{m}(K, H)\right)$ is completely reducible.

REMARK 4.7. After the first draft of this paper was written, we have been kindly informed by J. Hartwig that the complete reducibility of finite dimensional weight representations is also proved in his preprint [6] in a more general setting of ambiskew polynomial rings via a different approach.

REMARK 4.8. It might be interesting to study the decomposition of the product of two finite-dimensional irreducible weight representations.

REMARK 4.9. When $m=1$, the above results are obtained in [7] for the Drinfeld double of the positive part of $U_{q}\left(s l_{2}\right)$, and equivalently for the two-parameter quantum groups $U_{r, s}\left(s l_{2}\right)$ in [2].

\section{The Whittaker model for the center $Z\left(U_{q}\left(f_{m}(K, H)\right)\right)$}

Let $\mathfrak{g}$ be a finite-dimensional complex semisimple Lie algebra and $U(\mathfrak{g})$ be its universal enveloping algebra. The Whittaker model for the center of $U(\mathfrak{g})$ was first studied by Kostant in [10]. The Whittaker model for the center $Z(U(\mathfrak{g}))$ is defined via a nonsingular character of the nilpotent Lie subalgebra $\mathfrak{n}^{+}$of $\mathfrak{g}$. Using the Whittaker model, Kostant studied the structure of Whittaker modules of $U(\mathfrak{g})$ and many important results about Whittaker modules were obtained in [10]. Later on, Kostant's idea was further generalized by Lynch in [11] and by Macdowell in [12] to the case of singular characters of $\mathfrak{n}^{+}$, and similar results were proved to hold in these cases.

The obstacle of generalizing the Whittaker model to the quantized enveloping algebra $U_{q}(\mathfrak{g})$ with $\mathfrak{g}$ of higher ranks is that there is no nonsingular character existing for the positive part $\left(U_{q}(\mathfrak{g})\right)^{>0}$ of $U_{q}(\mathfrak{g})$ because of the quantum Serre relations. In order to overcome this difficulty, it was Sevostyanov who first recognised the usage of the topological version $U_{h}(\mathfrak{g})$ over $C[[h]]$ of quantum groups. Using a family of Coxeter realizations $U_{h}^{s_{\pi}}(\mathfrak{g})$ of the quantum group $U_{h}(\mathfrak{g})$ indexed by the Coxeter elements $s_{\pi}$, he was able to prove Kostant's results for $U_{h}(\mathfrak{g})$ in [15]. However, in the simplest case of $\mathfrak{g}=s l_{2}$, the quantum Serre relations are trivial, thus a direct approach should still work and this possibility has been worked out recently in [13].

In addition, it is reasonable to expect that the Whittaker model exists for most of the deformations of $U_{q}\left(s l_{2}\right)$. In this section, we show that there is such a Whittaker model for the center of $U_{q}\left(f_{m}(K, H)\right)$, and will study the Whittaker modules for $U_{q}\left(f_{m}(K, H)\right)$. We obtain analogous results as in [10] and [13]. For the reader's convenience, we present all the details here. Following the convention in [10], we shall use the term 'Whittaker modules' instead of 'Whittaker representations'.

5.1. The center $\boldsymbol{Z}\left(\boldsymbol{U}_{q}\left(f_{m}(K, \boldsymbol{H})\right)\right)$ of $\boldsymbol{U}_{q}\left(f_{m}(K, H)\right)$ In this subsection, we give a description of the center of $U_{q}\left(f_{m}(K, H)\right)$. The center $Z\left(U_{q}(f(K, H))\right)$ was also studied in [17] as well. As mentioned at the very beginning, we always assume that $f_{m}(K, H)=\left(K^{m}-H^{m}\right) /\left(q-q^{-1}\right)$ and $q$ is not a root of unity. 
We define a Casimir element of $U_{q}\left(f_{m}(H, K)\right)$ by setting

$$
\Omega=F E+\frac{q^{2 m} K^{m}+H^{m}}{\left(q^{2 m}-1\right)\left(q-q^{-1}\right)} .
$$

We have the following proposition.

PROPOSITION 5.1.

$$
\begin{aligned}
\Omega & =F E+\frac{q^{2 m} K^{m}+H^{m}}{\left(q^{2 m}-1\right)\left(q-q^{-1}\right)} \\
& =E F+\frac{K^{m}+q^{2 m} H^{m}}{\left(q^{2 m}-1\right)\left(q-q^{-1}\right)} .
\end{aligned}
$$

ProOF. Since $E F=F E+\left(\left(K^{m}-H^{m}\right) /\left(q-q^{-1}\right)\right.$,

$$
\begin{aligned}
\Omega & =F E+\frac{q^{2 m} K^{m}+H^{m}}{\left(q^{2 m}-1\right)\left(q-q^{-1}\right)} \\
& =E F-\frac{K^{m}-H^{m}}{q-q^{-1}}+\frac{q^{2 m} K^{m}+H^{m}}{\left(q^{2 m}-1\right)\left(q-q^{-1}\right)} \\
& =E F+\frac{K^{m}+q^{2 m} H^{m}}{\left(q^{2 m}-1\right)\left(q-q^{-1}\right)} .
\end{aligned}
$$

So we are done.

In addition, we have the following lemma.

LEMMA 5.2. $\Omega$ is in the center of $U_{q}\left(f_{m}(K, H)\right)$.

PROOF. It suffices to show that $\Omega E=E \Omega, \Omega F=F \Omega, \Omega K=K \Omega, \Omega H=H \Omega$. We shall only verify that $\Omega E=E \Omega$ and the rest of them are similar:

$$
\begin{aligned}
\Omega E & =\left(F E+\frac{q^{2 m} K^{m}+H^{m}}{\left(q^{2 m}-1\right)\left(q-q^{-1}\right)}\right) E \\
& =\left(E F-\frac{K^{m}-H^{m}}{q-q^{-1}}+\frac{q^{2 m} K^{m}+K^{-m}}{\left(q^{2 m}-1\right)\left(q-q^{-1}\right)}\right) E \\
& =E(F E)+\frac{K^{m}+q^{2 m} H^{m}}{\left(q-q^{-1}\right)\left(q^{2 m}-1\right)} E \\
& =E\left(F E+\frac{q^{2 m} K^{m}+H^{m}}{\left(q^{2 m}-1\right)\left(q-q^{-1}\right)}\right) \\
& =E \Omega .
\end{aligned}
$$

So we are done with the proof. 
In particular, we have the following description of the center $Z\left(U_{q}\left(f_{m}(K, H)\right)\right)$ of $U_{q}\left(f_{m}(K, H)\right)$.

Proposition 5.3 (see also [17]). $Z\left(U_{q}\left(f_{m}(K, H)\right)\right)$ is the subalgebra of $U_{q}\left(f_{m}(K, H)\right)$ generated by $\Omega,(K H)^{ \pm 1}$. In particular, $Z\left(U_{q}\left(f_{m}(K, H)\right)\right)$ is isomorphic to the localization $(\mathbb{C}[\Omega, K H])_{(K H)}$ of the polynomial ring in two variables $\Omega, K H$.

Proof. By Lemma 4.1, we have $\Omega,(K H)^{ \pm 1} \in Z\left(U_{q}\left(f_{m}(K, H)\right)\right)$. Thus the subalgebra $\mathbb{C}\left[\Omega,(K H)^{ \pm 1}\right]$ generated by $\Omega,(K H)^{ \pm 1}$ is contained in $Z\left(U_{q}\left(f_{m}(K, H)\right)\right)$. So it suffices to prove the other inclusion $Z\left(U_{q}\left(f_{m}(K, H)\right)\right) \subseteq \mathbb{C}\left[\Omega,(K H)^{ \pm 1}\right]$. Note that $U_{q}\left(f_{m}(K, H)\right)=\bigoplus_{n \in \mathbb{Z}_{>0}} U_{q}\left(f_{m}(K, H)\right)_{n}$ where $U_{q}\left(f_{m}(K, H)\right)_{n}$ is the $\mathbb{C}$-span of elements $\left\{u \in U_{q}\left(f_{m}(K, H)\right) \mid K u=q^{2 n} u K, H u=q^{-2 n} u H\right\}$. Suppose $x \in Z$ $\left(U_{q}\left(f_{m}(K, H)\right)\right)$, then $x K=K x, x H=H x$. Thus $x \in U_{q}\left(f_{m}(K, H)\right)_{0}$, which is generated by $E, F, K^{ \pm 1}, H^{ \pm 1}$. By the definition of $\Omega$, we know that $U_{q}\left(f_{m}(K, H)\right)_{0}$ is also generated by $\Omega, K^{ \pm 1}, H^{ \pm 1}$. Hence $x=\sum f_{i, j}(\Omega) K^{i} H^{j}$ where $f_{i, j}(\Omega)$ are polynomials in $\Omega$. Therefore

$$
x E=\sum f_{i, j}(\Omega) K^{i} H^{j} E=\sum f_{i, j}(\Omega) q^{2 i-2 j} E K^{i} H^{j}=E x,
$$

which forces $i=j$. So $x \in \mathbb{C}\left[\Omega,(K H)^{ \pm 1}\right]$ as desired. So we have proved that $Z\left(U_{q}\left(f_{m}(K, H)\right)\right)=\mathbb{C}\left[\Omega,(K H)^{ \pm 1}\right]$.

5.2. The Whittaker model for $Z\left(U_{q}\left(f_{m}(K, H)\right)\right)$ Now we construct the Whittaker model for $Z\left(U_{q}\left(f_{m}(K, H)\right)\right)$ following the lines in [10] and [13]. In the rest of this subsection, we shall denote the parameter Laurent polynomial $\left(K^{m}-H^{m}\right) /\left(q-q^{-1}\right)$ by $f(K, H)$ instead of $f_{m}(K, H)$.

First, we fix some notation. We denote by $U_{q}(E)$ the subalgebra of $U_{q}(f(K, H))$ generated by $E$, and by $U_{q}\left(F, K^{ \pm 1}, H^{ \pm 1}\right)$ the subalgebra of $U_{q}(f(K, H))$ generated by $F, K^{ \pm 1}, H^{ \pm 1}$. A nonsingular character of the algebra $U_{q}(E)$ can be defined as follows.

Definition 5.4. An algebra homomorphism $\eta: U_{q}(E) \longrightarrow \mathbb{C}$ is called a nonsingular character of $U_{q}(E)$ if $\eta(E) \neq 0$.

From now on, we shall fix such a nonsingular character of $U_{q}(E)$ and denote it by $\eta$. Following [10], we define the concepts of a Whittaker vector and a Whittaker module corresponding to the fixed nonsingular character $\eta$.

Definition 5.5. Let $V$ be a $U_{q}(f(K, H))$ module a vector $0 \neq v \in V$ is called a Whittaker vector of type $\eta$ if $E$ acts on $v$ through the nonsingular character $\eta$, that is, $E v=\eta(E) v$. If $V=U_{q}(f(K, H)) v$, then we call $V$ a Whittaker module of type $\eta$, and $v$ is called a cyclic Whittaker vector of type $\eta$.

The following decomposition of $U_{q}(f(K, H))$ is obvious. 
Proposition 5.6. $U_{q}(f(K, H))$ is isomorphic to $U_{q}\left(F, K^{ \pm 1}, H^{ \pm 1}\right) \otimes_{\mathbb{C}} U_{q}(E)$ as a vector space, and $U_{q}(f(K, H))$ is a free module over the subalgebra $U_{q}(E)$.

Let us denote the kernel of $\eta: U_{q}(E) \longrightarrow \mathbb{C}$ by $U_{q, \eta}(E)$, and we have the following decompositions of $U_{q}(E)$ and $U_{q}(f(K, H))$.

Proposition 5.7. We have $U_{q}(E)=\mathbb{C} \oplus U_{q, \eta}(E)$. In addition,

$$
U_{q}(f(K, H)) \cong U_{q}\left(F, K^{ \pm 1}, H^{ \pm 1}\right) \oplus U_{q}(f(K, H)) U_{q, \eta}(E) .
$$

PROOF. It is obvious that $U_{q}(E)=\mathbb{C} \oplus U_{q, \eta}(E)$. And

$$
U_{q}(f(K, H))=U_{q}\left(F, K^{ \pm 1}, H^{ \pm 1}\right) \otimes\left(\mathbb{C} \oplus U_{q, \eta}(E)\right),
$$

thus

$$
U_{q}(f(K, H)) \cong U_{q}\left(F, K^{ \pm 1}, H^{ \pm 1}\right) \oplus U_{q}(f(K, H)) U_{q, \eta}(E) .
$$

So we are done.

Now we define a projection

$$
\pi: U_{q}(f(K, H)) \longrightarrow U_{q}\left(F, K^{ \pm 1}, H^{ \pm 1}\right)
$$

from $U_{q}(f(K, H))$ onto $U_{q}\left(F, K^{ \pm 1}, H^{ \pm 1}\right)$ by taking the $U_{q}\left(F, K^{ \pm 1}, H^{ \pm 1}\right)$ component of any $u \in U_{q}(f(K, H))$. We denote the image $\pi(u)$ of $u \in U_{q}(f(K, H))$ as $u^{\eta}$ for short.

LEMMA 5.8. If $v \in Z\left(U_{q}(f(K, H))\right)$ and $u \in U_{q}(f(K, H))$, then we have $u^{\eta} v^{\eta}=(u v)^{\eta}$.

ProOF. Let $v \in Z\left(U_{q}(f(K, H))\right), u \in U_{q}(f(K, H))$, then

$$
\begin{aligned}
u v-u^{\eta} v^{\eta} & =\left(u-u^{\eta}\right) v+u^{\eta}\left(v-v^{\eta}\right) \\
& =v\left(u-u^{\eta}\right)+u^{\eta}\left(v-v^{\eta}\right)
\end{aligned}
$$

which is in $U_{q}(f(K, H)) U_{q, \eta}(E)$. Hence $(u v)^{\eta}=u^{\eta} v^{\eta}$.

By the definition of $\Omega$, we have the following description of $\pi(\Omega)$.

LEMMA 5.9.

$$
\pi(\Omega)=\eta(E) F+\frac{q^{2 m} K^{m}+H^{m}}{\left(q^{2 m}-1\right)\left(q-q^{-1}\right)} .
$$

Proposition 5.10. The map

$$
\pi: Z\left(U_{q}(f(K, H)) \longrightarrow U_{q}\left(F, K^{ \pm 1}, H^{ \pm 1}\right)\right.
$$

is an algebra isomorphism of $Z\left(U_{q}(f(K, H))\right)$ onto its image $W\left(F, K^{ \pm 1}, H^{ \pm 1}\right)$ in $U_{q}\left(F, K^{ \pm 1}, H^{ \pm 1}\right)$. 
PROOF. It follows from Lemma 5.8. that $\pi$ is a homomorphism of algebras. By Lemma 5.9,

$$
\pi(\Omega)=\eta(E) F+\frac{q^{2 m} K^{m}+H^{m}}{\left(q^{2 m}-1\right)\left(q-q^{-1}\right)}
$$

with $\eta(E) \neq 0$. Note that $\pi(K H)=K H$. We show that $\pi$ is injective. Suppose that $\pi(z)=0$ for some element $0 \neq z \in Z\left(U_{q}(f(K, H))\right)$. Since $Z\left(U_{q}(f(K, H))\right.$ $=\mathbb{C}\left[\Omega,(K H)^{ \pm 1}\right]$, we can write $z=\sum_{i=0}^{k} z_{i}(K H) \Omega^{i}$ where $z_{i}(K H)$ are nonzero Laurent polynomials in $\mathbb{C}\left[(K H)^{ \pm 1}\right]$. Since $\pi(z)=0$, then $u_{k}(K H)(\eta(E))^{k} F^{k}=0$, which is a contradiction. So $\pi$ is an injection. Thus $\pi$ is an algebra isomorphism from $Z\left(U_{q}(f(K, H))\right)$ onto its image $W\left(F, K^{ \pm 1}, H^{ \pm 1}\right)$ in $U_{q}\left(F, K^{ \pm 1}, H^{ \pm 1}\right)$.

LEMMA 5.11. If $u^{\eta}=u$, then

$$
u^{\eta} v^{\eta}=(u v)^{\eta}
$$

for any $v \in U_{q}(f(K, H))$.

PROOF. We have

$$
\begin{aligned}
u v-u^{\eta} v^{\eta} & =\left(u-u^{\eta}\right) v+u^{\eta}\left(v-v^{\eta}\right) \\
& =u^{\eta}\left(v-v^{\eta}\right),
\end{aligned}
$$

which is in $U_{q}(f(K, H)) U_{q, \eta}(E)$. So

$$
u^{\eta} v^{\eta}=(u v)^{\eta}
$$

for any $v \in U_{q}(f(K, H))$.

Let $\tilde{A}$ be the subspace of $U_{q}(f(K, H))$ spanned by $K^{ \pm i}$ where $i \in \mathbb{Z}_{\geq 0}$. Then $\tilde{A}$ is a graded vector space with

$$
\tilde{A}_{[n]}=\mathbb{C} K^{n} \oplus \mathbb{C} K^{-n}
$$

for $n \geq 1$, and

$$
\tilde{A}_{[0]}=\mathbb{C},
$$

and

$$
\tilde{A}_{[n]}=0
$$

for $n \leq-1$.

We define a filtration of $U_{q}\left(F, K^{ \pm 1}, H^{ \pm 1}\right)$ as follows:

$$
U_{q}\left(F, K^{ \pm 1}, H^{ \pm 1}\right)_{[n]}=\bigoplus_{i m+|j-k| \leq n m} U_{q}\left(F, K^{ \pm 1}, H^{ \pm 1}\right)_{i, j, k}
$$

with $U_{q}\left(F, K^{ \pm 1}, H^{ \pm 1}\right)_{i, j, k}$ being the vector space spanned by $F^{i} K^{j} H^{k}$.

We denote

$$
W\left(F, K^{ \pm 1}, H^{ \pm 1}\right)_{[p]}=\mathbb{C}\left[(K H)^{ \pm 1}\right]-\operatorname{span}\left\{1, \Omega^{\eta}, \ldots,\left(\Omega^{\eta}\right)^{p}\right\}
$$


for $q \geq 0$. It is easy to see that

$$
W\left(F, K^{ \pm 1}, H^{ \pm 1}\right)_{[p]} \subset W\left(F, K^{ \pm 1}, H^{ \pm 1}\right)_{[p+1]},
$$

and

$$
W\left(F, K^{ \pm 1}, H^{ \pm 1}\right)=\sum_{p \geq 0} W\left(F, K^{ \pm 1}, H^{ \pm 1}\right)_{[p]} .
$$

Note that $W\left(F, K^{ \pm 1}, H^{ \pm 1}\right)_{[p]}$ give a filtration of $W\left(F, K^{ \pm 1}, H^{ \pm 1}\right)$ which is compatible with the filtration of $U_{q}\left(F, K^{ \pm 1}, H^{ \pm 1}\right)$. In particular,

$$
W\left(F, K^{ \pm 1}, H^{ \pm 1}\right)_{[p]}=W\left(F, K^{ \pm 1}, H^{ \pm 1}\right) \cap U_{q}\left(F, K^{ \pm 1}, H^{ \pm 1}\right)_{[p]}
$$

for $p \geq 0$ via direct computations.

Now, we have a decomposition of $U_{q}\left(F, K^{ \pm 1}, H^{ \pm 1}\right)$ as follows.

THEOREM 5.12. $U_{q}\left(F, K^{ \pm 1}, H^{ \pm 1}\right)$ is free (as a right module) over $W\left(F, K^{ \pm 1}, H^{ \pm 1}\right)$. And the multiplication induces an isomorphism

$$
\Phi: \tilde{A} \otimes W\left(F, K^{ \pm 1}, H^{ \pm 1}\right) \longrightarrow U_{q}\left(F, K^{ \pm 1}, H^{ \pm 1}\right)
$$

as right $W\left(F, K^{ \pm 1}, H^{ \pm 1}\right)$ modules. In particular, we have the following formula:

$$
\bigoplus_{p+l m=n m} \tilde{A}_{[p]} \otimes W\left(F, K^{ \pm 1}, H^{ \pm 1}\right)_{[l]} \cong U_{q}\left(F, K^{ \pm 1}, H^{ \pm 1}\right)_{[n]} .
$$

Proof. Note that the map $\tilde{A} \times W\left(F, K^{ \pm 1}, H^{ \pm 1}\right) \longrightarrow U_{q}\left(F, K^{ \pm 1}, H^{ \pm 1}\right)$ is bilinear. So by the universal property of the tensor product, there is a map from $\tilde{A} \otimes W$ $\left(F, K^{ \pm 1}, H^{ \pm 1}\right)$ into $U_{q}\left(F, K^{ \pm 1}, H^{ \pm 1}\right)$ defined by the multiplication. It is easy to check that this map is a homomorphism of right $W\left(F, K^{ \pm 1}, H^{ \pm 1}\right)$ modules and is surjective as well.

Now, it remains to show that the map is injective. Let $0 \neq u \in \tilde{A} \otimes W$ $\left(F, K^{ \pm 1}, H^{ \pm 1}\right)$ with $\Phi(u)=0$. We can write

$$
u=\sum_{i=0}^{N} a_{i}(K) \otimes b_{i}(K H)\left(\eta(E) F+\frac{q^{2 m} K^{m}+H^{m}}{\left(q^{2 m}-1\right)\left(q-q^{-1}\right)}\right)^{i}
$$

where $u_{i}(K)$ are nonzero Laurent polynomials in $\mathbb{C}\left[K^{ \pm 1}\right]$ and $b_{i}(K H)$ are nonzero Laurent polynomials in $\mathbb{C}\left[(K H)^{ \pm 1}\right]$. Since $\Phi(u)=0$, then by direct computations,

$$
a_{N}(K) b_{N}(K H)(\eta(E))^{N} F^{N}=0 .
$$

Thus $a_{N}(K) b_{N}(K H)(\eta(E))^{N}=0$, which is a contradiction. So we have proved that $\Phi$ is indeed an isomorphism of vector spaces.

In addition, by counting the degrees of both sides, we also have

$$
\bigoplus_{p+l m=n m} \tilde{A}_{[p]} \otimes W\left(F, K^{ \pm 1}, H^{ \pm 1}\right)_{[l]} \cong U_{q}\left(F, K^{ \pm 1}, H^{ \pm 1}\right)_{[n]} .
$$

Thus the theorem is proved. 
Let $Y_{\eta}$ be the left $U_{q}(f(K, H))$ module defined by

$$
Y_{\eta}=U_{q}(f(K, H)) \otimes_{U_{q}(E)} \mathbb{C}_{\eta},
$$

where $\mathbb{C}_{\eta}$ is the one-dimensional $U_{q}(E)$ module defined by the character $\eta$. It is easy to see that

$$
Y_{\eta} \cong U_{q}(f(K, H)) / U_{q}(f(K, H)) U_{q, \eta}(E)
$$

is a Whittaker module with a cyclic vector denoted by $1_{\eta}$. Now we have a quotient map from $U_{q}(f(K, H))$ to $Y_{\eta}$ as follows:

$$
U_{q}(f(K, H)) \longrightarrow Y_{\eta} \quad \text { is defined by } u \mapsto u 1_{\eta},
$$

for any $u \in U_{q}(f(K, H))$.

If $u \in U_{q}(f(K, H))$, then there is a $u^{\eta}$ which is the unique element in $U_{q}\left(F, K^{ \pm 1}, H^{ \pm 1}\right)$ such that $u 1_{\eta}=u^{\eta} 1_{\eta}$. As in [10], we define the $\eta$-reduced action of $U_{q}(E)$ on $U_{q}\left(F, K^{ \pm 1}, H^{ \pm 1}\right)$ as follows:

$$
x \cdot v=(x v)^{\eta}-\eta(x) v,
$$

where $x \in U_{q}(E)$ and $v \in U_{q}\left(F, K^{ \pm 1}, H^{ \pm 1}\right)$.

LemMA 5.13. Let $u \in U_{q}(f(K, H))$ and $x \in U_{q}(E)$,

$$
x \cdot u^{\eta}=[x, u]^{\eta} .
$$

PROOF. $[x, u] 1_{\eta}=(x u-u x) 1_{\eta}=(x u-\eta(x) u) 1_{\eta}$. Hence

$$
[x, u]^{\eta}=(x u)^{\eta}-\eta(x) u^{\eta}=\left(x u^{\eta}\right)^{\eta}-\eta(x) u^{\eta}=x \cdot u^{\eta} .
$$

LemmA 5.14. Let $x \in U_{q}(E), u \in U_{q}\left(F, K^{ \pm 1}, H^{ \pm 1}\right)$, and $v \in W\left(E, K^{ \pm 1}, H^{ \pm 1}\right)$, then

$$
x \cdot(u v)=(x \cdot u) v
$$

ProOF. Let $v=w^{\eta}$ for some $w \in Z\left(U_{q}(f(K, H))\right.$, then $u v=u w^{\eta}=u^{\eta} w^{\eta}$ $=(u w)^{\eta}$. Thus

$$
\begin{aligned}
x \cdot(u v) & =x \cdot(u w)^{\eta}=[x, u w]^{\eta} \\
& =([x, u] w)^{\eta}=[x, u]^{\eta} w^{\eta} \\
& =\left(x \cdot u^{\eta}\right) v \\
& =(x \cdot u) v .
\end{aligned}
$$

So we are done. 
Let $V$ be a $U_{q}(f(K, H))$ module and let $U_{q, V}(f(K, H))$ be the annihilator of $V$ in $U_{q}(f(K, H))$. Then $U_{q, V}(f(K, H))$ defines a central ideal $Z_{V} \subset Z\left(U_{q}(f(K, H))\right)$ by setting $Z_{V}=U_{q, V}(f(K, H)) \cap Z\left(U_{q}(f(K, H))\right)$. Suppose that $V$ is a Whittaker module with a cyclic Whittaker vector $w$, and we denote by $U_{q, w}(f(K, H))$ the annihilator of $w$ in $U_{q}(f(K, H))$. It is obvious that

$$
U_{q}(f(K, H)) U_{q, \eta}(E)+U_{q}(f(K, H)) Z_{V} \subset U_{q, w}(f(K, H)) .
$$

In the next theorem, we show that the reverse inclusion holds. First of all, we need an auxiliary lemma.

Lemma 5.15. Let $X=\left\{v \in U_{q}\left(F, K^{ \pm 1}, H^{ \pm 1}\right) \mid(x \cdot v) w=0, x \in U_{q}(E)\right\}$. Then

$$
X=\tilde{A} \otimes W_{V}\left(F, K^{ \pm 1}, H^{ \pm 1}\right)+W\left(F, K^{ \pm 1}, H^{ \pm 1}\right),
$$

where $W_{V}\left(F, K^{ \pm 1}, H^{ \pm 1}\right)=\left(Z_{V}\right)^{\eta}$. In fact, $U_{q, V}\left(F, K^{ \pm 1}, H^{ \pm 1}\right) \subset X$ and

$$
U_{q, w}\left(F, K^{ \pm 1}, H^{ \pm 1}\right)=\tilde{A} \otimes W_{w}\left(F, K^{ \pm 1}, H^{ \pm 1}\right),
$$

where $U_{q, w}\left(F, K^{ \pm 1}, H^{ \pm 1}\right)=U_{q, w}(f(K, H)) \cap U_{q}\left(F, K^{ \pm 1}, H^{ \pm 1}\right)$.

PROOF. Let us denote by $Y=\tilde{A} \otimes W_{V}\left(F, K^{ \pm 1}, H^{ \pm 1}\right)+W\left(F, K^{ \pm 1}, H^{ \pm 1}\right)$ where $W\left(F, K^{ \pm 1}, H^{ \pm 1}\right)=\left(Z\left(U_{q}(f(K, H))\right)\right)^{\eta}$. Thus we need to verify $X=Y$. Let $v \in W\left(F, K^{ \pm 1}, H^{ \pm 1}\right)$, then $v=u^{\eta}$ for some $u \in Z\left(U_{q}(f(K, H))\right)$. So

$$
\begin{aligned}
x \cdot v & =x \cdot u^{\eta} \\
& =[x, u]^{\eta} \\
& =(x u)^{\eta}-\eta(x) u^{\eta} \\
& =x^{\eta} u^{\eta}-\eta(x) u^{\eta} \\
& =0 .
\end{aligned}
$$

So we have $W\left(F, K^{ \pm 1}, H^{ \pm 1}\right) \subset X$. Let $u \in Z_{V}$ and $v \in U_{q}\left(F, K^{ \pm 1}, H^{ \pm 1}\right)$. Then for any $x \in U_{q}(E)$,

$$
x \cdot\left(v u^{\eta}\right)=(x \cdot v) u^{\eta} .
$$

Since $u \in Z_{V}$, then $u^{\eta} \in U_{q, w}(f(K, H))$. Thus we have $v u^{\eta} \in X$, and hence

$$
\tilde{A} \otimes W_{V}\left(F, K^{ \pm 1}, H^{ \pm 1}\right) \subset X,
$$

which proves $Y \subset X$. Note that $\tilde{\left.A_{[i}\right]}$ is the subspace of $\mathbb{C}\left[K^{ \pm 1}\right]$ spanned by $K^{ \pm i}$, and let $\overline{W_{V}\left(F, K^{ \pm 1}, H^{ \pm 1}\right)}$ be the complement of $W_{V}\left(F, K^{ \pm 1}, H^{ \pm 1}\right)$ in $W\left(F, K^{ \pm 1}, H^{ \pm 1}\right)$.

Let us set

$$
M_{i}=\tilde{A_{[i]}} \otimes \overline{W_{V}\left(F, K^{ \pm 1}, H^{ \pm 1}\right)},
$$


and thus we have the following formula:

$$
U_{q}\left(F, K^{ \pm 1}, H^{ \pm 1}\right)=M \oplus Y
$$

where $M=\sum_{i \geq 1} M_{i}$. We show that $M \cap X \neq 0$. Let $M_{[k]}=\sum_{1 \leq i \leq k} M_{i}$, and then $M_{[k]}$ are a filtration of $M$. Suppose $n$ is the smallest integer such that $X \cap M_{[n]}$ $\neq 0$ and $0 \neq y \in X \cap M_{[n]}$. Then we have $y=\sum_{1 \leq i \leq n} y_{i}$ where $y_{i} \in \tilde{A}_{i} \otimes$ $\overline{W_{V}\left(F, K^{ \pm 1}, H^{ \pm 1}\right)}$. Suppose we have chosen $y$ in such a way that $y$ has the fewest terms. By similar computations as used in [13], we have $0 \neq y-1 /\left(\eta(E)\left(q^{-2 n}-1\right)\right)$ $E \cdot y \in X \cap M_{[n]}$ with fewer terms than $y$. This is a contradiction. So we have $X \cap M=0$.

Now we prove that $U_{q, w}\left(F, K^{ \pm 1}, H^{ \pm 1}\right) \subset X$. Let $u \in U_{q, w}\left(F, K^{ \pm 1}, H^{ \pm 1}\right)$ and $x \in U_{q}(E)$, then we have $x u w=0$ and $u x w=\eta(x) u w=0$. Thus $[x, u] \in U_{q, w}$ $(f(K, H))$, and hence $[x, u]^{\eta} \in U_{q, w}\left(F, K^{ \pm 1}, H^{ \pm 1}\right)$. Since $u \in U_{q, w}\left(F, K^{ \pm 1}, H^{ \pm 1}\right)$ $\subset U_{q, w}\left(E, F, K^{ \pm 1}, H^{ \pm 1}\right)$, then $x \cdot u=[x, u]^{\eta}$. Thus $x \cdot u \in U_{q, w}\left(F, K^{ \pm 1}, H^{ \pm 1}\right)$. So $u \in X$ by the definition of $X$. Now we are going to prove the following formula:

$$
W\left(F, K^{ \pm 1}, H^{ \pm 1}\right) \cap U_{q, w}\left(F, K^{ \pm 1}, H^{ \pm 1}\right)=W_{V}\left(F, K^{ \pm 1}, H^{ \pm 1}\right) .
$$

In fact, $W_{V}\left(F, K^{ \pm 1}, H^{ \pm 1}\right)=\left(Z_{V}^{\eta}\right)$ and $W_{V}\left(F, K^{ \pm 1}, H^{ \pm 1}\right) \subset U_{q, w}\left(F, K^{ \pm 1}, H^{ \pm 1}\right)$. So if $v \in W_{w}\left(F, K^{ \pm 1}, H^{ \pm 1}\right) \cap U_{q, w}\left(F, K^{ \pm 1}, H^{ \pm 1}\right)$, then we can uniquely write $v=u^{\eta}$ for $u \in Z\left(U_{q}(f(K, H))\right)$. Then $v w=0$ implies $u w=0$ and hence $u \in Z$ $\left(U_{q}(f(K, H))\right) \cap U_{q, w}\left(F, K^{ \pm 1}, H^{ \pm 1}\right)$. Since $V$ is generated cyclically by $w$, we have proved the above statement. Obviously, we have $U_{q}(f(K, H)) Z_{V} \subset U_{q, w}$ $(f(K, H))$. Thus we have $\tilde{A} \otimes W_{V}\left(F, K^{ \pm 1}, H^{ \pm 1}\right) \subset U_{q, w}\left(F, K^{ \pm 1}, H^{ \pm 1}\right)$. Therefore,

$$
U_{q, w}\left(F, K^{ \pm 1}, H^{ \pm 1}\right)=\tilde{A} \otimes W_{V}\left(F, K^{ \pm 1}, H^{ \pm 1}\right) .
$$

So we have finished the proof.

THEOREM 5.16. Let $V$ be a Whittaker module admitting a cyclic Whittaker vector $w$, then we have

$$
U_{q, w}(f(K, H))=U_{q}(f(K, H)) Z_{V}+U_{q}(f(K, H)) U_{q, \eta}(E) .
$$

PROOF. It is obvious that

$$
U_{q}(f(K, H)) Z_{V}+U_{q}(f(K, H)) U_{\eta}(E) \subset U_{q, w}(f(K, H)) .
$$

Let $u \in U_{q, w}(f(K, H))$, and we show that $u \in U_{q}(f(K, H)) Z_{V}+U_{q}(f(K, H))$ $U_{q, \eta}(E)$. Let $v=u^{\eta}$, and then it suffices to show that $v \in \tilde{A} \otimes W_{V}\left(F, K^{ \pm 1}, H^{ \pm 1}\right)$. But $v \in U_{q, w}\left(F, K^{ \pm 1}, H^{ \pm 1}\right)=\tilde{A} \otimes W_{V}\left(F, K^{ \pm 1}, H^{ \pm 1}\right)$. So we have proved the theorem. 
THEOREM 5.17. Let $V$ be any Whittaker module for $U_{q}(f(K, H))$, then the correspondence

$$
V \mapsto Z_{V}
$$

sets up a bijection between the set of all equivalence classes of Whittaker modules and the set of all ideals of $Z\left(U_{q}(f(K, H))\right)$.

PROOF. Let $V_{i}, i=1,2$ be two Whittaker modules. If $Z_{V_{1}}=Z_{V_{2}}$, then clearly $V_{1}$ is equivalent to $V_{2}$ by the above theorem. Now, let $Z_{*}$ be an ideal of $Z\left(U_{q}(f(K, H))\right)$ and let $L=U_{q}(f(K, H)) Z_{*}+U_{q}(f(K, H)) U_{\eta}(E)$. Then $V=U_{q}(f(K, H)) / L$ is a Whittaker module with a cyclic Whittaker vector $w=\overline{1}$. Obviously, we have $U_{q, w}(f(K, H))=L$. So $L=U_{q, w}(f(K, H))$ $=U_{q}(f(K, H)) Z_{V}+U_{q}(f(K, H)) U_{q, \eta}(E)$. This implies that

$$
\pi\left(Z_{*}\right)=\pi(L)=\pi\left(Z_{V}\right) .
$$

Since $\pi$ is injective on $Z\left(U_{q}(f(K, H))\right)$, thus $Z_{V}=Z_{*}$. Thus we have finished the proof.

THEOREM 5.18. Let $V$ be a $U_{q}(f(K, H))$ module. Then $V$ is a Whittaker module if and only if

$$
V \cong U_{q}(f(K, H)) \otimes_{Z\left(U_{q}(f(K, H))\right) \otimes U_{q}(E)}\left(Z\left(U_{q}(f(K, H))\right) / Z_{*}\right)_{\eta} .
$$

In particular, in such a case, the ideal $Z_{*}$ is uniquely determined to be $Z_{V}$.

PROOF. If $1_{*}$ is the image of 1 in $Z\left(U_{q}(f(K, H))\right) / Z_{*}$, then

$$
\operatorname{Ann}_{Z\left(U_{q}(f(K, H))\right) \otimes U_{q}(F)}\left(1_{*}\right)=U_{q}(E) Z_{*}+Z\left(U_{q}(f(K, H))\right) U_{q, \eta}(E) .
$$

Thus the annihilator of $w=1 \otimes 1_{*}$ is

$$
U_{q, w}(f(K, H))=U_{q}(f(K, H)) Z_{*}+U_{q}(f(K, H)) U_{q, \eta}(E) .
$$

Then the result follows from the previous theorem.

THEOREM 5.19. Let $V$ be a $U_{q}(f(K, H))$ module with a cyclic Whittaker vector $w \in V$. Then any $v \in V$ is a Whittaker vector if and only if $v=u w$ for some $u \in Z\left(U_{q}(f(K, H))\right)$.

PROOF. If $v=u w$ for some $u \in Z\left(U_{q}(f(K, H))\right)$, then it is obvious that $v$ is a Whittaker vector. Conversely, let $v=u w$ for some $u \in U_{q}(f(K, H))$ be a Whittaker vector of $V$. Then $v=u^{\eta} w$ by the definition of Whittaker module. So we may assume that $u \in U_{q}\left(F, K^{ \pm 1}, H^{ \pm 1}\right)$. If $x \in U_{q}(E)$, then we have $x u w=\eta(x) u w$ and $u x w=\eta(x) u w$. Thus $[x, u] w=0$ and hence $[x, u]^{\eta} w=0$. But we have $x \cdot u=[x, u]^{\eta}$. Thus we have $u \in X$. We can now write $u=u_{1}+u_{2}$ with $u_{1} \in U_{q, w}$ $\left(F, K^{ \pm 1}, H^{ \pm 1}\right)$ and $u_{2} \in W\left(F, K^{ \pm 1}, H^{ \pm 1}\right)$. Then $u_{1} w=0$. Hence $u_{2} w=v$. But $u_{2}=u_{3}^{\eta}$ with $u_{3} \in Z\left(U_{q}(f(K, H))\right)$. So we have $v=u_{3} w$ which proves the theorem. 
Now let $V$ be a Whittaker module and $\operatorname{End}_{U_{q}(f(K, H))}(V)$ be the endomorphism ring of $V$ as a $U_{q}(f(K, H))$ module. Then we can define the following homomorphism of algebras using the action of $Z\left(U_{q}(f(K, H))\right)$ on $V$ :

$$
\pi_{V}: Z\left(U_{q}(f(K, H)) \longrightarrow \operatorname{End}_{U_{q}(f(K, H))}(V) .\right.
$$

It is clear that

$$
\begin{aligned}
& Z\left(U_{q}(f(K, H))\right) / Z_{V}\left(U_{q}(f(K, H))\right) \cong \pi_{V}\left(Z\left(U_{q}(f(K, H))\right)\right) \\
& \quad \subset \operatorname{End}_{U_{q}(f(K, H))}(V) .
\end{aligned}
$$

In fact, the next theorem says that this inclusion is an equality as well.

THEOREM 5.20. Let $V$ be a Whittaker $U_{q}(f(K, H))$ module. Then $\operatorname{End}_{U_{q}(f(K, H))}(V)$ $\cong Z\left(U_{q}(f(K, H))\right) / Z_{V}$. In particular, $\operatorname{End}_{U_{q}(f(K, H))}(V)$ is commutative.

Proof. Let $w \in V$ be a cyclic Whittaker vector. If $\alpha \in \operatorname{End}_{U_{q}(f(K, H))}(V)$, then $\alpha(w)=u w$ for some $u \in Z\left(U_{q}(f(K, H))\right)$ by Theorem 5.19. Thus we have $\alpha(v w)=v u w=u v w$. Hence $\alpha=\pi_{u}$, which proves the theorem.

Now we are going to construct explicitly some Whittaker modules. Let

$$
\xi: Z\left(U_{q}(f(K, H))\right) \longrightarrow \mathbb{C}
$$

be a central character of the center $Z\left(U_{q}(f(K, H))\right)$. For any given central character $\xi$, let $Z_{\xi}=\operatorname{Ker}(\xi) \subset Z\left(U_{q}(f(K, H))\right)$ and $Z_{\xi}$ is a maximal ideal of $Z\left(U_{q}(f(K, H))\right)$. Since $\mathbb{C}$ is algebraically closed, then $Z_{\xi}=\left(\Omega-a_{\xi}, K H-b_{\xi}\right)$ for some $a_{\xi} \in \mathbb{C}, b_{\xi} \in \mathbb{C}^{*}$. For any given central character $\xi$, let $\mathbb{C}_{\xi, \eta}$ be the onedimensional $Z\left(U_{q}(f(K, H))\right) \otimes U_{q}(E)$ module defined by $u v y=\xi(u) \eta(v) y$ for any $u \in Z\left(U_{q}(f(K, H))\right)$ and any $v \in U_{q}(E)$. We set

$$
Y_{\xi, \eta}=U_{q}(f(K, H)) \otimes_{Z\left(U_{q}(f(K, H))\right) \otimes U_{q}(E)} \mathbb{C}_{\xi, \eta} .
$$

It is easy to see that $Y_{\xi, \eta}$ is a Whittaker module of type $\eta$ and admits a central character $\xi$. By Schur's lemma, we know that every irreducible representation has a central character. As studied in [17], we know that $U_{q}(f(K, H))$ has a similar theory for Verma modules. In fact, Verma modules also fall into the category of Whittaker modules if we take the trivial character of $U_{q}(E)$. Namely we have the following formula:

$$
M_{\lambda}=U_{q}(f(K, H)) \otimes_{U_{q}\left(E, K^{ \pm 1}, H^{ \pm 1}\right)} \mathbb{C}_{\lambda},
$$

where $K, H$ act on $\mathbb{C}_{\lambda}$ through the character $\lambda$ of $\mathbb{C}\left[K^{ \pm 1}, H^{ \pm 1}\right]$, and $U_{q}(E)$ act trivially on $\mathbb{C}_{\lambda}$. Thus, $M_{\lambda}$ admits a central character. It is well known that Verma modules may not necessarily be irreducible, even though they have central characters. However, Whittaker modules are at the other extreme, as shown in the next theorem.

THEOREM 5.21. Let $V$ be a Whittaker module for $U_{q}(f(K, H))$. Then the following statements are equivalent. 
(1) $V$ is irreducible.

(2) $V$ admits a central character.

(3) $Z_{V}$ is a maximal ideal.

(4) The space of Whittaker vectors of $V$ is one-dimensional.

(5) All nonzero Whittaker vectors of $V$ are cyclic.

(6) The centralizer $\operatorname{End}_{U_{q}(f(K, H))}(V)$ is reduced to $\mathbb{C}$.

(7) $V$ is isomorphic to $Y_{\xi, \eta}$ for some central character $\xi$.

PROOF. It is easy to see that (2)-(7) are equivalent to each other by using the previous theorems that we have just proved. Since $\mathbb{C}$ is algebraically closed and uncountable, we also know that (1) implies (2) by using a theorem due to Dixmier [3]. To complete the proof, it suffices to show that (2) implies (1), namely that if $V$ has a central character, then $V$ is irreducible.

Let $\omega \in V$ be a cyclic Whittaker vector, then $V=U_{q}(f(K, H)) \omega$. We have $V=U_{q}\left(F, K^{ \pm 1}, H^{ \pm 1}\right) w$. Since $V$ is irreducible, then $V$ has a central character. Thus we have $\Omega w=\lambda(\Omega) w$. Now

$$
\Omega w=\left(\eta(E) F+\frac{q^{2 m} K^{m}+H^{m}}{\left(q^{2 m}-1\right)\left(q-q^{-1}\right)}\right) w .
$$

Hence the action of $F$ on $V$ is uniquely determined by the action of $K$ and $H$ on $V$, and $H^{-1} v=a K v, K^{-1} v=b H v$ for some $a, b \in \mathbb{C}^{*}$ and for any $v \in V$. Thus $V$ has a $\mathbb{C}$-basis consisting of elements $\left\{K^{i} \omega, H^{j} \omega \mid i, j \in \mathbb{Z}_{\geq 0}\right\}$.

Let

$$
0 \neq v=\left(\sum_{i=0}^{n} a_{i} K^{i}+\sum_{j=1}^{m} b_{j} H^{j}\right) \omega \in V,
$$

then

$$
\begin{aligned}
E\left(\sum_{i=0}^{n} a_{i} K^{i}+\sum_{j=1}^{m} b_{j} H^{j}\right) \omega & =\left(\sum_{i=0}^{n} q^{-2 i} a_{i} K^{i}+\sum_{j=1}^{m} q^{2 j} b_{j} H^{j}\right) E \omega \\
& =\eta(E)\left(\sum_{i=0}^{n} q^{-2 i} a_{i} K^{i}+\sum_{j=1}^{m} q^{2 j} b_{j} H^{j}\right) \omega .
\end{aligned}
$$

Thus we have $0 \neq \eta(E) q^{-2 n} v-E v \in V$, in which the top degree of $K$ is $n-1$. By repeating this operation finitely many times, we shall finally get an element $0 \neq a \omega$ with $a \in \mathbb{C}^{*}$. This means that $V=U_{q}(f(K, H)) v$ for any $0 \neq v \in V$. So $V$ is irreducible. Therefore, we are done with the proof.

In addition, the proof of the previous theorem also implies the following result.

THEOREM 5.22. Let $(V, w)$ be an irreducible Whittaker module with a Whittaker vector $w$, then $V$ has a $\mathbb{C}$-basis consisting of elements $\left\{K^{i} \omega, H^{j} \omega \mid i, j \in \mathbb{Z}_{\geq 0}\right\}$.

It is easy to show the following two theorems, but for more details about the proof, we refer the reader to [10]. 
THEOREM 5.23. Let $V$ be a $U_{q}(f(K, H))$ module which admits a central character. Assume that $w \in V$ is a Whittaker vector. Then the submodule $U_{q}(f(K, H)) w \subset V$ is irreducible.

THEOREM 5.24. Let $V_{1}, V_{2}$ be any two irreducible $U_{q}(f(K, H))$ modules with the same central character. If $V_{1}$ and $V_{2}$ contain Whittaker vectors, then these vectors are unique up to scalars. Furthermore, $V_{1}$ and $V_{2}$ are isomorphic to each other as $U_{q}(f(K, H))$ modules.

\section{References}

[1] V. V. Bavula, 'Generalized Weyl algebras and their representations', Algebra i Analiz 4(1) (1992), 75-97; (Engl. Transl. St Petersburg Math. J. 4 (1993) 71-93).

[2] G. Benkart and S. Witherspoon, 'Representations of two-parameter quantum groups and Shur-Weyl duality', in: Hopf Algebras, Lecture Notes in Pure and Applied Mathematics, 237 (Dekker, New York, 2004), pp. 65-92.

[3] J. Dixmier, Enveloping Algebras (North-Holland, Amsterdam, 1977).

[4] V. G. Drinfeld, 'Hopf algebras and the quantum Yang-Baxter equations', Soviet math. Dokll 32 (1985), 254-258.

[5] P. Gabriel, 'Des categories abeliennes', Bull. Soc. Math. France 90 (1962), 323-449.

[6] J. Hartwig, 'Hopf structures on ambiskew polynomial rings', J. Pure Appl. Algebra 212(4) (2008), 863-883.

[7] J. Hu and Y. Zhang, 'Quantum double of $U_{q}\left(\left(s l_{2}\right)^{\leq 0}\right)$ ', J. Algebra 317(1) (2007), 87-110.

[8] Q. Ji, D. Wang and X. Zhou, 'Finite dimensional representations of quantum groups $U_{q}(f(K))$ ', East-West J. Math. 2(2) (2000), 201-213.

[9] N. Jing and J. Zhang, 'Quantum Weyl algebras and deformations of $U(G)$ ', Pacific J. Math. 171(2) (1995), 437-454.

[10] B. Kostant, 'On Whittaker vectors and representation theory', Invent. Math. 48(2) (1978), 101-184.

[11] T. Lynch, 'Generalized Whittaker vectors and representation theory', PhD Thesis, MIT, 1979.

[12] E. Macdowell, 'On modules induced from Whittaker modules', J. Algebra 96 (1985), 161-177.

[13] M. Ondrus, 'Whittaker modules for $U_{q}\left(s l_{2}\right)^{\text {', }}$ J. Algebra 289 (2005), 192-213.

[14] A. Rosenberg, Noncommutative Algebraic Geometry and Representations of Quantized Algebras, Mathematics and its Applications, 330 (Kluwer Academic Publishers, 1995).

[15] A. Sevostyanov, 'Quantum deformation of Whittaker modules and Toda lattice', Duke Math. J. 204(1) (2000), 211-238.

[16] X. Tang, 'Construct irreducible representations of quantum groups $U_{q}\left(f_{m}(K)\right)$ ', Front. Math. China 3(3) (2008), 371-397.

[17] D. Wang, Q. Ji and S. Yang, 'Finite-dimensional representations of quantum group $U_{q}(f(K, H))$ ', Comm. Algebra 30 (2002), 2191-2211.

\section{XIN TANG, Department of Mathematics \& Computer Science, Fayetteville State University, Fayetteville, NC 28301, USA e-mail: xtang@uncfsu.edu}

YUNGE XU, Faculty of Mathematics \& Computer Science, Hubei University, Wuhan 430062, People's Republic of China e-mail: xuy@hubu.edu.cn 